\title{
MC study for the effect of diffractive events on air shower developments
}

\author{
Ken Ohashi ${ }^{* 1}$, Hiroaki Menjo ${ }^{1}$, Yoshitaka Itow ${ }^{1,2}$ \\ ${ }^{1}$ Institute for Space-Earth Environmental Research, Nagoya University, Nagoya, Japan \\ ${ }^{2}$ Kobayashi-Maskawa Insttute, Nagoya University, Nagoya, Japan \\ E-mail: ohashi.kendisee.nagoya-u.ac.jp
}

The origin of ultra-high energy cosmic rays is unknown and the mass composition is one of the key observables to understand the origin. The mass composition is estimated by comparing a prediction of the depth of maximum of shower developments, $X_{\max }$, with experimental data, however, the $X_{\max }$ prediction depends on the choice of hadronic interaction modes in the simulation. One of the proposed sources of the difference is the different modeling of diffractive collisions among the models. In this work, we estimate the effect of detail of diffractive collisions at the first interaction of cosmic-rays with atmospheric nuclei on the air shower developments by using the air shower simulation package COSMOS 8.035. The results show that the modeling of diffractive collisions at the first interaction is not the main source of the model discrepancies of the $X_{\max }$ prediction.

The 4th KMI International Symposium (KMI2019)

18-20, February 2019

Nagoya, Japan

\footnotetext{
* Speaker.
} 


\section{Introduction}

The origin of ultra-high energy cosmic rays (UHECRs) is unknown. To understand the origin, the mass composition of cosmic rays, which is a species of cosmic rays such as protons, iron nuclei, or other nuclei, is one of the key observables. The depth of maximum of the air shower developments, $X_{\max }$, is one of the indicators of the mass composition, and the mass composition is estimated by comparing the experimental data of $X_{\max }$ with the prediction. However, a prediction of $X_{\max }$ depends on the choice of hadronic interaction models in the simulation, and that makes difficult to interpret the mass composition. Therefore, experimental verification of hadronic interaction models is needed. Several components of the models are proposed as sources of discrepancies of the $X_{\max }$ predictions among the models. One proposed source is the different modeling of diffractive collisions among the models.

The diffractive collision is one of the event categories of collisions between hadrons, and $16 \%$ to $22 \%$ of collisions between a $10^{15} \mathrm{eV}$ proton and an air nucleus are diffractive collisions. There are four types of diffractive collisions. A single diffractive (SD) event is a diffractive collision with one particle dissociation, while a double diffractive (DD) event is with both particles dissociations. The first interaction in air shower is the interaction between a cosmic-ray proton and an air nucleus, so SD events can be divided into two types, with projectile proton dissociation (projectile $\mathrm{SD}$ ) and with target nucleus dissociation (target SD). The other category is the central diffraction (CD). In this study, collision types other than diffractive collisions are called the non-diffraction (ND). Diffractive collisions are characterized by a smaller number and higher energies of produced particles than non-diffractive collisions. Figure $\mathbb{W}$ is the schematic view of the interaction of these categories.

A few simulation studies about the effect of diffractive collisions on the air-shower development have been performed. The effect of the shift of diffractive cross section on $X_{\max }$ is estimated $\pm 5 \mathrm{~g} / \mathrm{cm}^{2}$ [四]. In another work [0], the maximum effect of diffractive collisions is estimated with an extreme assumption, and that is $15 \mathrm{~g} / \mathrm{cm}^{2}$ at maximum. However, the assumption of that work is not realistic that the air showers without diffractive collisions are considered, and the effect of details of diffractive collisions on $X_{\max }$ is not well understood. It is important to understand the effect of details of diffractive collisions on the $X_{\max }$ predictions for improvements of the models. In this work, we discuss the effect of diffractive collisions on the mean $X_{\max }\left(<X_{\max }>\right)$. As the first step to understanding the effect, we focus on collisions at the first hadronic interaction between a cosmic-ray and an atmospheric nucleus.

\section{Monte Carlo Simulation}

In this work, we simulate air showers by using the air shower simulation package COSMOS 8.035 [B] , and estimate the effect of details of diffractive collisions on mean $X_{\max }\left(\left\langle X_{\max }\right\rangle\right)$. In the air shower simulation, one of three hadronic interaction models, EPOS-LHC [四], QGSJET II04 []], and SIBYLL 2.3c [G, 四] is used for hadronic interactions above $80 \mathrm{GeV}$, and DPMJET III [8] and phits [Q] are used for hadronic interactions with $2-80 \mathrm{GeV}$ and less than $2 \mathrm{GeV}$, respectively. 50,000 air showers with $10^{15} \mathrm{eV}$ proton incidence are simulated for each hadronic interaction model and categorize them by the collision type at the first hadronic interaction between a cosmic- 


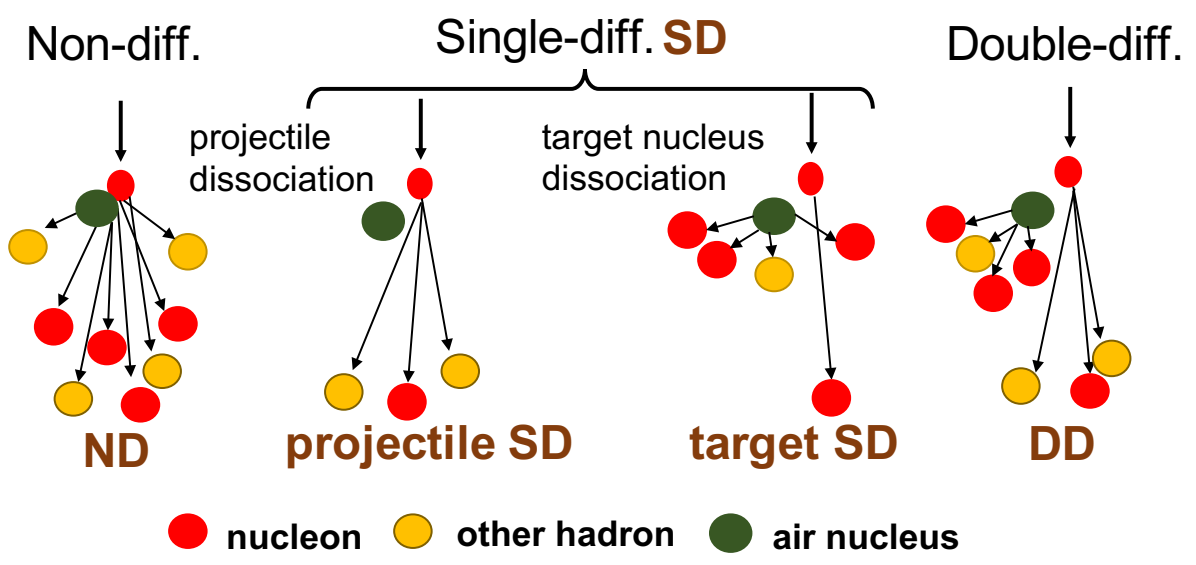

Figure 1: The schematic view of the interaction between a cosmic-ray proton and an air nucleus. The collision type is non-diffractive (left), single diffraction with projectile proton dissociation (middle-left), single diffraction with target air nucleus dissociation (middle-right), and double diffraction (right).

Table 1: the predictions of $\left\langle X_{\max }\right\rangle$ and fractions of each category with statistical errors for three hadronic interaction models.

\begin{tabular}{cc|cccccc}
\hline & & $\mathrm{ND}$ & projectile SD & target SD & $\mathrm{DD}$ & $\mathrm{CD}$ & total \\
\hline \multirow{3}{*}{ SIBYLL 2.3c } & fraction [\%] & $84.2 \pm 0.4$ & $10.5 \pm 0.1$ & $4.20 \pm 0.09$ & $1.10 \pm 0.05$ & & 100.0 \\
& $<\mathrm{X}_{\max }>\left[\mathrm{g} / \mathrm{cm}^{2}\right]$ & $577.0 \pm 0.4$ & $609.9 \pm 1.3$ & $648.1 \pm 2.5$ & $605.7 \pm 3.8$ & $583.8 \pm 0.4$ \\
QGSJET II-04 & fraction [\%] & $84.7 \pm 0.4$ & $7.2 \pm 0.1$ & $4.20 \pm 0.09$ & $4.00 \pm 0.09$ & & 100.0 \\
& $<\mathrm{X}_{\max }>\left[\mathrm{g} / \mathrm{cm}^{2}\right]$ & $561.1 \pm 0.4$ & $612.4 \pm 1.6$ & $634.8 \pm 2.5$ & $602.8 \pm 2.0$ & $569.9 \pm 0.4$ \\
EPOS-LHC & fraction [\%] & $78.9 \pm 0.4$ & $4.7 \pm 0.1$ & $5.0 \pm 0.1$ & $9.2 \pm 0.1$ & $2.27 \pm 0.07$ & 100.0 \\
\hline
\end{tabular}

ray and an atmospheric nucleus. For saving the calculation time, we use the one-dimensional approximation for electromagnetic showers initiated by electrons or photons with energies less than $0.01 E$, where $E$ is the energy of the primary proton. The number of electrons is calculated each 25 $\mathrm{g} / \mathrm{cm}^{2}$, and $X_{\max }$ is calculated by fitting them with the Gaisser-Hillas function. Definitions of the collision type are different among the hadronic interaction models. We use the same definition of the collision type as in the previous work [[]].

\section{The effect of the diffractive collisions on the air shower developments}

The simulated events are divided into the four or five categories by using the collision type at the first interaction, and $\left\langle X_{\max }\right\rangle$ are calculated for each category. Table 1 shows the $\left\langle X_{\max }\right\rangle$ and fractions of each category. The $\left\langle X_{\max }\right\rangle$ predictions are different among the categories. The $<X_{\max }>$ predictions of the categories of diffractive collisions are larger than that of ND, and that of target SD are largest in the four or five categories. The predictions of $\left\langle X_{\max }\right\rangle$ and the fraction of each category are different among the hadronic interaction models. Not only the predictions of $\left\langle X_{\max }\right\rangle$ but also the fraction of each category affects to the $\left\langle X_{\max }\right\rangle$ predictions for all events because the $\left\langle X_{\max }>\right.$ predictions are different among categories. In the next sections, we focus on the effect of differences of the predictions of $\left\langle X_{\max }\right\rangle$ and the fraction of each category among hadronic interaction models on the air shower developments. 
Table 2: the predictions of $\left\langle X_{\max }^{N D}>, \alpha^{i}\right.$ and fractions of each category. $\alpha^{i}$ is defined in Equation 1.2.

\begin{tabular}{ll|ccccc}
\hline & & $\mathrm{ND}$ & projectile SD & target SD & DD & $\mathrm{CD}$ \\
\hline \multirow{3}{*}{ SIBYLL 2.3c } & fraction [\%] & 84.2 & 10.5 & 4.2 & 1.1 & \\
& $\mathrm{X}_{\max }^{\mathrm{ND}}$ or $\alpha^{i}$ & 577.0 & +32.9 & +71.1 & +28.7 & \\
& fraction [\%] & 84.7 & 7.2 & 4.2 & 3.9 & \\
QGSJET II-04 & $\mathrm{X}_{\max }^{\mathrm{ND}}$ or $\alpha^{i}$ & 561.1 & +50.9 & +73.3 & +41.3 & \\
& fraction [\%] & 78.9 & 4.7 & 5.0 & 9.2 & 2.3 \\
EPOS-LHC & $\mathrm{X}_{\max }^{\mathrm{ND}}$ or $\alpha^{i}$ & 565.5 & +45.6 & +67.3 & +40.6 & +61.6 \\
\hline
\end{tabular}

\subsection{The effect of the fraction of each type of diffraction}

To estimate the effect of model discrepancies in the fraction, we replace fractions in QGSJET II to those in EPOS-LHC or SIBYLL 2.3c, and calculate the shift of total $X_{\max }$ by using Eq. B.] and 3.2.

$$
\begin{gathered}
<X_{\max }^{\text {total }}>=<X_{\max }^{N D}>+f^{\text {projectileSD }} \alpha^{\text {projectileSD }}+f^{\text {target } S D} \alpha^{\text {target } S D}+f^{D D} \alpha^{D D} \\
<\mathrm{X}_{\max }^{i}>=<\mathrm{X}_{\max }^{\mathrm{ND}}>+\alpha^{i}
\end{gathered}
$$

where $\left\langle X_{\max }^{\text {total }}\right\rangle$ is $\left\langle X_{\max }>\right.$ of all events, and $\left\langle X_{\max }^{i}\right\rangle$ is that of the i category. The label i means one of the categories. $f^{i}$ is the fraction of each category. $\alpha^{i}$ is defined in Eq. B.2 and is a difference of $<X_{\max }>$ between the category i from ND. This $\alpha^{i}$ is introduced for the analysis in the next section. Only EPOS-LHC has the category of CD. In replacements, the fraction of CD is ignored and each fraction other than CD is renormalized in order to make the total fraction $100 \%$. When $\left\langle X_{\max }^{\text {total }}\right\rangle$ is calculated with $\left\langle X_{\max }^{N D}>\right.$ and $\alpha^{i}$ in QGSJET II-04 and $f^{i}$ in SIBYLL 2.3c by using Eq. B.D, which is the replacement of the fractions in QGSJET II-04 by SIBYLL 2.3c, the result is $570.4 \mathrm{~g} / \mathrm{cm}^{2}$ and that is $0.5 \mathrm{~g} / \mathrm{cm}^{2}$ larger than the original prediction by QGSJET II. The result of replacements of the fraction in QGSJET II by EPOS-LHC is $571.6 \mathrm{~g} / \mathrm{cm}^{2}$, which is $1.7 \mathrm{~g} / \mathrm{cm}^{2}$ larger than the original prediction by QGSJET II. From these results, the effect of model differences of the fraction between SIBYLL 2.3c (EPOS-LHC) and QGSJET II on $\left\langle X_{\max }>\right.$ is $0.5 \mathrm{~g} / \mathrm{cm}^{2}\left(1.7 \mathrm{~g} / \mathrm{cm}^{2}\right)$, which is $3.5 \%(12.2 \%)$ of the current size of model discrepancy in the $\left\langle X_{\max }^{\text {total }}\right\rangle$ predictions among the models.

\subsection{The effect of different predictions of particle production in each diffractive type among models}

The model discrepancies in the prediction of $\left\langle X_{\max }\right\rangle$ of each category of diffractive collisions are caused by both the difference of predictions of particle productions in diffractive collisions and that in non-diffractive collisions, and we separate these two by an ad-hoc way. To separate the effects, we only replace $\alpha^{i}$ of each category between the models while $\left\langle X_{\max }^{N D}>\right.$ is not replaced, and this is way $\alpha^{i}$ is introduced in the previous section.

The effect of different predictions of particle production in each diffractive type among models is estimated by replacing $\alpha^{i}$ in QGSJET II-04 by that in another model and calculating $\left\langle X_{\max }^{\text {total }}\right\rangle$ by using Eq. T.]. The result of the replacement of $\alpha^{i}$ in QGSJET II-04 by SIBYLL 2.3c (EPOS-LHC) is $568.0 \mathrm{~g} / \mathrm{cm}^{2}\left(569.2 \mathrm{~g} / \mathrm{cm}^{2}\right)$, which is $1.9 \mathrm{~g} / \mathrm{cm}^{2}\left(0.6 \mathrm{~g} / \mathrm{cm}^{2}\right)$ smaller than original predictions by QGSJET II-04. The size of this shift is $13.6 \%$ (4.3\%) of the current model discrepancy in $\left\langle X_{\max }^{\text {total }}\right\rangle$ predictions among the models. 


\section{Conclusion}

In this work, the effects of the differences of predictions of the particle production and the fraction of each type of diffraction among models on the $\left\langle X_{\max }\right\rangle$ prediction are estimated by using collision type at the first hadronic interaction between a cosmic-ray and an atmospheric nucleus, and the size of these effects are $13.6 \%$ and $12.2 \%$ of the current model discrepancy in $\left\langle X_{\max }\right\rangle$ predictions between models, respectively. These effects are not negligible, however, they are not the main source of the current model discrepancy.

In the estimation of the effects in this work, air showers are simulated with the relatively small energy of the incidence proton than UHECRs and only the collision type of the first interaction are considered. For the next step, we try to estimate the effects with higher energy and with considering other interactions in the air shower. Moreover, to understand the effect of the different modeling of each diffractive type among models more precisely, we also try to estimate the effects by using the diffractive mass, which is an invariant mass of the diffractive dissociation system and an important parameter for particle productions of the diffractive collision.

\section{References}

[1] S.Ostapchenko, LHC data on inelastic diffraction and uncertainties in the predictions for longitudinal extensive air shower development, Physical Review D 89 (2014) 074009.

[2] L. B. Arbeletche, V. P. Goncalves, and M. A.Muller, Investigating the influence of diffractive interactions on ultra - high energy extensive air showers, International Journal of Modern Physics A 33 (26) 01.

[3] K. Kasahara et al., https://cosmos.n.kanagawa-u.ac.jp/cosmosHome/index.html

[4] T. Pierog, I. Karpenko, J. M. Katzy, E. Yatsenko, and K. Werner, EPOS LHC: Test of collective hadronization with data measured at the CERN Large Hadron Collider, Physical Review C 92 (2015) 034906 .

[5] S. Ostapchenko, Monte Carlo treatment of hadronic interactions in enhanced Pomeron scheme: I. QGSJET-II model, Physical Review D 83 (2011) 014018.

[6] E.-J. Ahn, R. Engel, T. K. Gaisser, P. Lipari, and T. Stanev, Cosmic ray interaction event generator SIBYLL 2.1, Physical Review D 80 (2009) 094003.

[7] F. Riehn et al., The hadronic interaction model Sibyll $2.3 \mathrm{c}$ and Feynman scaling, PoS ICRC2017 (2017) 301 .

[8] S. Roesler, R. Engel, and J. Ranft, http://sroesler.web.cern.ch/sroesler/dpmjet3.html

[9] T. Sato et al., Features of Particle and Heavy Ion Transport code System (PHITS) version 3.02, Journal of Nuclear Science and Technology 55 (2018) 684-690. 